Bull. Austral. Math. Soc.

VOL. $53(1996) \quad[91-100]$

\title{
CRITICAL ALGEBRAS AND THE FRATTINI CONGRUENCE, II
}

\section{KeITH A. KeARNES}

\begin{abstract}
We prove that any finite subdirectly irreducible algbra in a congruence modular variety with trivial Frattini congruence is critical. We also show that if $\mathbf{A}$ and $\mathbf{B}$ are critical algebras which generate the same congruence modular variety, then the variety generated by the proper sections of $A$ equals the variety generated by the proper sections of $\mathbf{B}$.
\end{abstract}

\section{INTRODUCTION}

An algebra $\mathbf{C}$ is a section of $\mathbf{A}$ if there is a subalgebra $\mathbf{B} \leqslant \mathbf{A}$ and a surjective homomorphism $\varphi: \mathbf{B} \rightarrow \mathbf{C}$. $\mathbf{C}$ is a proper section unless $\mathbf{B}=\mathbf{A}$ and $\varphi$ is an isomorphism. The class of proper sections of $A$ is denoted (HS-1)A. A is critical if it is finite and does not belong to the subvariety generated by its proper sections.

Problem 25 of H. Neumann's book [8] asks the following question: If $\mathbf{A}$ and $\mathbf{B}$ are critical groups generating the same variety, must the varieties generated by (HS - 1)A and (HS - 1)B be the same? Macdonald had previously shown in [6] that the answer is affirmative if $\mathbf{A}$ and $\mathbf{B}$ are $p$-groups. Assuming that $\mathcal{V}$ is generated by a critical $p$ group $\mathbf{C}$, Macdonald showed how to construct equations axiomatising $\operatorname{var}((\mathbf{H S}-1) \mathbf{C})$ relative to $\mathcal{V}$ solely from the fact that $\mathcal{V}$ is generated by a critical $p$-group. His construction of equations works the same way for $\mathbf{A}$ or $\mathbf{B}$ when $\mathcal{V}=\operatorname{var}(\mathbf{A})=\operatorname{var}(\mathbf{B})$, so the result follows. Using properties of the Frattini subgroup, Bryant gave an affirmative answer to Neumann's question for arbitrary finite groups in [1]. The same question for other types of algebras is considered in the papers $[9,7,10]$ and $[5]$. The last of these, by Kiss and Vovsi, subsumes the others with respect to this question. In their paper, Kiss and Vovsi prove that if $\mathbf{A}$ and $\mathbf{B}$ are critical algebras such that $\mathcal{V}:=\operatorname{var}(\mathbf{A})=\operatorname{var}(\mathbf{B})$ is congruence permutable, then the varieties generated by (HS - 1)A and (HS - 1)B are the same. Their proof is an elaboration of Bryant's argument. An interesting aspect of [5] is that the proof given seems to show that the 'correct' generalisation of the Frattini subgroup is a Frattini congruence, not a Frattini subalgebra.

This note is a sequel to the Kiss-Vovsi paper. The first part proves, with simpler arguments, a far broader result than any of those mentioned. Let $\mathbf{A}$ and $\mathbf{B}$ be critical algebras generating the same variety. We give an easy necessary and sufficient condition

Received 21 March 1995

Copyright Clearance Centre, Inc. Serial-fee code: 0004-9729/96 \$A2.00+0.00. 
for (HS - 1)A and (HS - 1)B to generate the same variety. From these conditions it is possible to deduce that when $\mathbf{A}$ and $\mathbf{B}$ are simple then (HS - 1) A and (HS - 1)B do generate the same variety. Weak local modularity hypotheses on $\mathbf{A}$ and $\mathbf{B}$ suffice to force (HS - 1)A and (HS - 1)B to generate the same variety. It will follow that if $\mathcal{V}:=\operatorname{var}(\mathbf{A})=\operatorname{var}(\mathbf{B})$ is congruence modular, then (HS - 1)A and (HS - 1)B generate the same variety. This solves a problem raised in [5].

The Kiss-Vovsi definition of the Frattini congruence is re-introduced in Section 3, along with a concept from [2] which I call the normalisation of a subdirectly irreducible algebra. If $\mathbf{A}$ is a finite subdirectly irreducible algebra with $\mathbf{A b e l i a n}$ monolith and $\mathbf{A}$ generates a congruence modular variety, then it turns out that $\mathbf{A}$ is isomorphic to its normalisation if and only if its Frattini congruence is trivial. Any such algebra is critical.

\section{VARIETIES GENERATED BY PROPER SECTIONS}

Let $(\mathbf{S}-1) \mathbf{A}$ denote the class of all proper subalgebras of $\mathbf{A}$ and $(\mathbf{H}-1) \mathbf{A}$ denote the class of all proper homomorphic images of $\mathbf{A}$. A finite algebra $\mathbf{A}$ is $\mathbf{S}$-critical or $\mathbf{H}$-critical if it is not a member of the variety generated by $(\mathbf{S}-1) \mathbf{A}$ or $(\mathbf{H}-\mathbf{1}) \mathbf{A}$, respectively. Any critical algebra is both $\mathbf{S}$-critical and $\mathbf{H}$-critical and any $\mathbf{H}$-critical algebra is subdirectly irreducible.

The following simple lemma allows us to avoid introducing the Frattini congruence in our discussion of Neumann's problem.

LEmMA 2.1. If $\mathbf{A}$ and $\mathrm{B}$ are $\mathrm{S}$-critical and generate the same variety, then $(\mathbf{S}-1) \mathbf{A}$ and $(\mathbf{S}-1) \mathbf{B}$ generate the same variety.

Proof: Let $\mathcal{V}$ denote $\operatorname{var}(A)=\operatorname{var}(B)$ and set $\mathcal{Q}=\operatorname{SP}(A) \cap \operatorname{SP}(B) . \mathcal{Q}$ is a quasivariety which is contained in $\mathcal{V}$ and which contains the free algebras of $\mathcal{V}$. Since $\mathcal{V}$ is finitely generated, $\mathcal{Q}$ contains a finite relatively free algebra which generates $\mathcal{V}$. Choose $C$ to be a member of $\mathcal{Q}$ which generates $\mathcal{V}$ and has least cardinality for this property.

Claim. $(\mathbf{S}-1) \mathbf{C}$ and $(\mathbf{S}-1) \mathbf{A}$ generate the same variety.

Proof of ClaIM: By the minimality hypothesis on $\mathbf{C}$, any proper subalgebra of $\mathbf{C}$ generates a proper subvariety of $\mathcal{V}$. Hence, no proper subalgebra of $\mathbf{C}$ has a homomorphism onto $A$. Since all subalgebras of $\mathbf{C}$ belong to $\mathbf{S P}(A)$, it follows that $(\mathbf{S}-1) \mathbf{C} \subseteq \mathbf{S P}((\mathbf{S}-1) \mathbf{A})$. Hence $(\mathbf{S}-1) \mathbf{C}$ is contained in $\operatorname{var}((\mathbf{S}-1) \mathbf{A})$. Conversely, since $\mathbf{C}$ generates $\mathcal{V}, \mathbf{C} \in \mathbf{S P}(\mathbf{A})$ and $(\mathbf{S}-1) \mathbf{A}$ does not generate $\mathcal{V}$, it follows that $\mathbf{C}$ has a homomorphism onto $\mathbf{A}$. If $h: \mathbf{C} \rightarrow \mathbf{A}$ is onto and $\mathbf{A}^{\prime}$ is a proper subalgebra of $\mathbf{A}$, then $\mathbf{C}^{\prime}:=h^{-1}\left(\mathbf{A}^{\prime}\right)$ is a proper subalgebra of $\mathbf{C}$ which has a homomorphism onto $\mathbf{A}^{\prime}$. Hence, $(\mathbf{S}-1) \mathbf{A}$ is contained in $\operatorname{var}((\mathbf{S}-1) \mathbf{C})$. We get that 
$\operatorname{var}((\mathbf{S}-1) \mathbf{A})=\operatorname{var}((\mathbf{S}-1) \mathbf{C})$.

It follows from the Claim that $\operatorname{var}((\mathbf{S}-1) \mathbf{A})=\operatorname{var}((\mathbf{S}-1) \mathbf{C})=\operatorname{var}((\mathbf{S}-1) \mathbf{B})$. This proves the lemma.

Lemma 2.1 corresponds to one half of Proposition 1 of $[5]$ in the case that $\operatorname{var}(\mathbf{A})=$ $\operatorname{var}(B)$ is a congruence permutable variety. In the other half of Proposition 1 of [5] it is proved that when $\mathbf{A}$ and $\mathbf{B}$ are $\mathbf{S}$-critical algebras in a congruence permutable variety and $\operatorname{var}(A)=\operatorname{var}(B)$, then $A / \Phi_{A} \cong B / \Phi_{B}$, where $\Phi$ denotes the Frattini congruence (defined in the next section). This other half of Proposition 1 can also be extended to arbitrary varieties by using the idea of the proof of Lemma 2.1. (In particular, if $\mathbf{A}$ and $\mathbf{B}$ are $\mathbf{S}$-critical algebras which generate the same variety and their Frattini congruences are trivial, then $\mathbf{A} \cong \mathbf{B}$.)

If an algebra $\mathbf{A}$ is critical, it is subdirectly irreducible. Denote its monolith by $\mu_{\mathbf{A}}$.

ThEOREM 2.2. Let $\mathbf{A}$ and $\mathbf{B}$ be critical algebras which generate the same variety. The inclusion

$$
\operatorname{var}((\mathbf{H S}-1) \mathbf{A}) \subseteq \operatorname{var}((\mathbf{H S}-1) \mathbf{B})
$$

holds if and only if

$$
\mathbf{A} / \mu_{\mathbf{A}} \in \operatorname{var}((\mathbf{H S}-1) \mathbf{B}) \text {. }
$$

Proof: Notice that $(\mathbf{H S}-1) \mathbf{A}=\mathbf{H}[(\mathbf{S}-1) \mathbf{A}] \cup(\mathbf{H}-1) \mathbf{A}$. Lemma 2.1 guarantees the equality in

$$
\mathbf{H}[(\mathbf{S}-1) \mathbf{A}] \subseteq \operatorname{var}((\mathbf{S}-1) \mathbf{A})=\operatorname{var}((\mathbf{S}-1) \mathbf{B}) \subseteq \operatorname{var}((\mathbf{H S}-1) \mathbf{B}) .
$$

Therefore, the inclusion

$$
\operatorname{var}((\mathbf{H S}-1) \mathbf{A}) \subseteq \operatorname{var}((\mathbf{H S}-1) \mathbf{B})
$$

holds if and only if

$$
(\mathbf{H}-1) \mathbf{A} \subseteq \operatorname{var}((\mathbf{H S}-1) \mathbf{B}) .
$$

But the condition $(\mathbf{H}-1) \mathbf{A} \subseteq \operatorname{var}((\mathbf{H S}-1) B)$ is equivalent to

$$
\mathbf{A} / \mu_{\mathbf{A}} \in \operatorname{var}((\mathbf{H S}-1) \mathbf{B}) \text {. }
$$

It follows from this theorem that critical algebras $\mathbf{A}$ and $\mathbf{B}$ which generate the same variety have the property that

$$
\operatorname{var}((\mathbf{H S}-1) \mathbf{A})=\operatorname{var}((\mathbf{H S}-1) \mathbf{B})
$$

if and only if $\mathbf{A} / \mu_{\mathbf{A}} \in \operatorname{var}((\mathbf{H S}-1) \mathbf{B})$ and $\mathbf{B} / \mu_{\mathbf{B}} \in \operatorname{var}((\mathbf{H S}-1) \mathbf{A})$. 
Corollary 2.3. If $\mathrm{A}$ and $\mathrm{B}$ are simple critical algebras which generate the same variety, then (HS - 1)A and (HS - 1)B generate the same variety.

The Kiss-Vovsi result can be extended from congruence permutable varieties to congruence modular varieties by simply combining Theorem 2.2 with their Proposition 2. Let us see how to extend it further still.

If $\mathbf{A}$ is a finite algebra and $\alpha \prec \beta$ in $\operatorname{Con}(\mathbf{A})$, then the prime quotient $\langle\alpha, \beta\rangle$ will be called modular if the $\langle\alpha, \beta\rangle$-minimal sets are of type 2,3 or 4 and these minimal sets have empty tails. If the $\langle\alpha, \beta\rangle$-minimal sets are of type 3 or 4 and these minimal sets have empty tails, then $\langle\alpha, \beta\rangle$ is distributive. Modular and distributive quotients have the following nice properties. Assume that $\mathbf{A}^{\prime}$ is finite, $h: \mathbf{A}^{\prime} \rightarrow \mathbf{A}$ is onto and that $\langle\alpha, \beta\rangle$ is a prime quotient of $\mathbf{A}$. Set $\alpha^{\prime}=h^{-1}(\alpha)$ and $\beta^{\prime}=h^{-1}(\beta)$. If $\langle\alpha, \beta\rangle$ is modular (distributive), then

(i) $\left\langle\alpha^{\prime}, \beta^{\prime}\right\rangle$ is modular (distributive), and

(ii) there is a homomorphism of $\operatorname{Con}\left(\mathbf{A}^{\prime}\right)$ onto a modular (distributive) lattice which separates $\alpha^{\prime}$ and $\beta^{\prime}$.

In particular, it follows that if all prime quotients of $\mathbf{A}$ are modular (distributive), then Con $(A)$ is a modular (distributive) lattice. For stronger results, Theorems 8.5 and 8.6 of [3] can be rephrased to say that a locally finite variety $\mathcal{V}$ is congruence modular (distributive) if and only if all prime quotients of finite mumbers of $\mathcal{V}$ are modular (distributive).

LemmA 2.4. Assume that $\mathbf{A}$ and $\mathbf{B}$ are critical algebras which generate the same variety and that $\left\langle 0, \mu_{\mathbf{A}}\right\rangle$ is distributive. Then $\mathbf{A} \cong \mathbf{B}$ and so $\operatorname{var}((\mathbf{H S}-1) \mathbf{A})=$ $\operatorname{var}((\mathbf{H S}-1) \mathbf{B})$.

Proof: If $\left\langle 0, \mu_{\mathbf{A}}\right\rangle$ is distributive and $\mathbf{A} \in \operatorname{var}(\mathbf{B})$, then a local version of Jónsson's Lemma proves that $\mathbf{A} \in \mathbf{H S}(\mathbf{B})$. Since $\mathbf{A} \notin(\mathbf{H S}-1) \mathbf{B}$, it must be that $\mathbf{A} \cong \mathbf{B}$

For the next theorem, a congruence $\theta$ on a finite algebra is hereditarily modular if each prime quotient $\langle\alpha, \beta\rangle$ with $0 \leqslant \alpha \prec \beta \leqslant \theta$ is modular.

Theorem 2.5. Assume that $\mathbf{A}$ and $\mathrm{B}$ are critical algebras which generate the same variety and that $\left\langle 0, \mu_{\mathbf{A}}\right\rangle$ with $\left\langle 0, \mu_{\mathbf{B}}\right\rangle$ are modular. If every Abelian congruence on either $\mathbf{A}$ or $\mathbf{B}$ is hereditarily modular, then $\operatorname{var}((\mathbf{H S}-1) \mathbf{A})=\operatorname{var}((\mathbf{H S}-1) \mathbf{B})$.

Proof: Assume the hypotheses of the theorem, but that $\operatorname{var}((\mathbf{H S}-1) \mathrm{A}) \nsubseteq$ $\operatorname{var}((\mathbf{H S}-1) B)$. By Lemma 2.4, it must be that $\operatorname{typ}\left(0, \mu_{A}\right)=\operatorname{typ}\left(0, \mu_{B}\right)=2$. Since $\mathbf{A} \in \operatorname{var}(\mathbf{B})$, there is a finite algebra $\mathbf{C}$ which is a subdirect product of subdirectly irreducible algebras from $\mathbf{H S}(\mathbf{B})$ for which there is an onto homomorphism $h: \mathbf{C} \rightarrow \mathbf{A}$. Since $C$ is a subdirect product of subdirectly irreducible algebras from HS(B), $C$ has meet-irreducible congruences $\eta_{i}, i<n$, such that $\mathbf{C} / \eta_{i} \in \mathbf{H S}(\mathrm{B})$. For each $i$, let $\eta_{i}^{*}$ 
denote the unique upper cover of $\eta_{i}$. Let $\delta=\operatorname{ker} h$ and let $\delta^{*}$ be its unique upper cover. Let $\theta$ be the least congruence on $\mathbf{C}$ such that $\mathbf{C} / \theta \in \operatorname{var}((\mathbf{H S}-1) \mathrm{B})$. Clearly, $\theta \leqslant \eta_{i}^{*}$ for all $i$ while $\theta \not \eta_{i}$ holds if and only if $\mathbf{C} / \eta_{i} \cong \mathbf{B}$. By rearranging indices if necessary, we may assume that $\theta \not \eta_{i}$ for $i<j$ and that $\theta \leqslant \eta_{i}$ for $j \leqslant i<n$. (This implies that $\mathrm{C} / \eta_{i} \cong \mathrm{B}$ for $i<j$, in which case $\left\langle\eta_{i}, \eta_{i}^{*}\right\rangle$ is modular of type 2.) Since

$$
0_{\mathbf{B}}=\bigwedge_{i<n} \eta_{i} \leqslant \theta \leqslant\left(\bigwedge_{i<j} \eta_{i}^{*}\right) \wedge\left(\bigwedge_{j \leqslant i<n} \eta_{i}\right)
$$

and $\operatorname{typ}\left(\eta_{i}, \eta_{i}^{*}\right)=2$ for $i<j$, it must be that $\theta$ is Abelian. If $\theta \leqslant \delta^{*}$, then

$$
\mathbf{A} / \mu_{\mathbf{A}} \cong \mathbf{C} / \delta^{*} \in \mathbf{H}(\mathbf{C} / \theta) \subseteq \operatorname{var}((\mathbf{H S}-1) \mathbf{B}) \text {. }
$$

But if this were so, then Theorem 2.2 would force $\operatorname{var}((\mathbf{H S}-1) \mathrm{A}) \subseteq \operatorname{var}((\mathbf{H S}-1) \mathrm{B})$ which contradicts the assumption in the first sentence of this proof. Hence $\theta \leq \delta^{*}$ and so $\delta^{*}<\delta \vee \theta$. Since $\theta$ is Abelian, $\delta$ is meet-irreducible and $\left\langle\delta, \delta^{*}\right\rangle$ is modular of type 2, the interval $I[\delta, \delta \vee \theta]$ is Abelian. It follows that $h(\delta \vee \theta)$ is an Abelian congruence of A. By hypothesis, $h(\delta \vee \theta)$ is hereditarily modular. Therefore, every prime quotient in the interval $I[\delta, \delta \vee \theta]$ of $\operatorname{Con}(C)$ is modular.

The following comparabilities and non-comparabilities in $\operatorname{Con}(\mathbf{C})$ have been established:

$$
\begin{aligned}
& \text { (i) } 0=\bigwedge_{i<n} \eta_{i} \leqslant \theta \leqslant \bigwedge_{i<n} \eta_{i}^{*} . \\
& \text { (ii) } \theta \leqslant \eta_{i} \text { for all } j \leqslant i<n . \\
& \text { (iii) } \theta \not \eta_{i} \text { for } i<j . \\
& \text { (iv) } \theta \not \leq \delta^{*} .
\end{aligned}
$$

Con $(\mathbf{C})$ has a homomorphism onto a modular lattice which separates all modular prime quotients. Such a homomorphism preserves all the comparabilities listed, of course. It also preserves the listed non-comparabilities, since $\left\langle\eta_{i}, \eta_{i}^{*}\right\rangle$ is modular for $i<j$ and every prime quotient in the interval $[\delta, \delta \vee \theta]$ is modular. We may henceforth assume that $\operatorname{Con}(\mathrm{C})$ is a modular lattice, as long as we depend only the comparabilities and non-comparabilities listed in this paragraph.

Let $\lambda=\bigwedge_{i<n} \eta_{i}^{*}$. By modularity, we have $\left(\eta_{i} \wedge \lambda\right) \prec \lambda$ whenever $\lambda \npreceq \eta_{i}$. Since $\bigwedge_{i<n} \eta_{i}=0$, the zero congruence is a meet of lower covers of $\lambda$. Therefore, the interval $I[0, \lambda]$ is a complemented modular lattice; hence $I[0 \vee \delta, \lambda \vee \delta]$ is a complemented modular lattice. But $\delta$ is meet-irreducible, so we must have $\lambda \vee \delta \leqslant \delta^{*}$. Since $\theta \leqslant \lambda$, this gives us the contradiction that $\theta \leqslant \delta^{*}$.

Theorem 2.5 solves the problem raised in [5] since, when $\operatorname{var}(A)=\operatorname{var}(B)$ is congruence modular, then all prime quotients of $\mathrm{A}$ and $\mathrm{B}$ are modular. 
We mention that there are critical algebras such that $\operatorname{var}(\mathbf{A})=\operatorname{var}(\mathbf{B})$ but for which $\operatorname{var}(($ HS -1$) A) \neq \operatorname{var}(($ HS -1$) B)$. Such examples can be easily constructed where $\operatorname{var}(A)=\operatorname{var}(B)$ is a variety of $G$-sets.

\section{Normalisation}

In this section we discuss a process called 'normalisation' which converts a subdirectly irreducible algebra (in a congruence modular variety) into a better-behaved and related algebra. This process is described in [2], but not named.

We shall follow the notation of [2] except in the following cases: First, when $R$ is a binary relation on $S$ we shall write $S \times{ }_{R} S \times_{R} \ldots \times_{R} S$, with $n$ factors, to denote the subset of $S^{n}$ which consists of the tuples $\left(s_{1}, \ldots, s_{n}\right)$ with $\left(s_{i}, s_{i+1}\right) \in R$. If $\mathrm{A}$ is an algebra and $\alpha$ is a congruence, we use boldface notation $\mathbf{A} \times_{\alpha} \cdots \times_{\alpha} \mathbf{A}$ to indicate the subalgebra of $\mathbf{A}^{n}$ supported by $A \times_{\alpha} \cdots \times_{\alpha} A$. (This notation differs from [2] in the following way: what we write as $\mathbf{A} \times_{\alpha} \mathbf{A}$ is denoted by $\mathbf{A}(\alpha)$ in [2].) Next, we shall write $\widehat{A}$ in this section for something which is denoted $D(\mathbf{A})$ in [2]. Finally, if $\mathbf{A}$ is an algebra, $\mathbf{B}$ is a subalgebra and $\boldsymbol{\theta}$ is a congruence on $\mathbf{A}$, then $\mathbf{B}^{\boldsymbol{\theta}}$ denotes the subalgebra of $\mathbf{A}$ whose universe is $\{x \in A \mid \exists y \in B((x, y) \in \theta)\}$. (This notion does not occur in [2].)

DEFinition 3.1: Assume that $\mathbf{A}$ is a subdirectly irreducible algebra with monolith $\mu$. The normalisation of $\mathbf{A}$ is defined as follows: if $\mu$ is nonabelian, then the normalisation of $\mathbf{A}$ is $\mathbf{A}$; if $\mu$ is Abelian, then the normalisation of $\mathbf{A}$ is

$$
\widehat{A}:=\left(\mathbf{A} \times_{\mu} \mathbf{A}\right) / \Delta_{\mu,(0: \mu)} .
$$

The congruence $\left(\mu_{0}+\Delta_{\mu,(0: \mu)}\right) / \Delta_{\mu,(0: \mu)}$ on $\widehat{A}$ is denoted $\widehat{\mu} . \quad \mathbf{A}$ is normal if it is isomorphic to its normalisation.

The next lemma summarises those properties of the normalisation which are proved in [2].

LEMмA 3.2: Let $\mathcal{K}$ be a finite set of finite algebras and $\mathbf{A}$ be a subdirectly irreducible algebra. Assume that $\operatorname{var}(\mathcal{K})$ and $\operatorname{var}(\mathbf{A})$ are congruence modular. Let $\mu$ be the monolith of $\mathbf{A}$.

(i) $\widehat{A}$ is a normal subdirectly irreducible algebra and $\hat{\mu}$ is its monolith.

(ii) $\mathbf{A} /(0: \mu) \cong \widehat{A} /(0: \widehat{\mu})$.

(iii) $(0: \widehat{\mu})=\widehat{\mu}$.

(iv) $\hat{\mu}$ is the kernel of a retraction.

(v) If $\mathbf{A} \in \operatorname{var}(\mathcal{K})$, then $\hat{A}$ is isomorphic to the normalisation of some subdirectly irreducible algebra in $\mathbf{H S}(\mathcal{K})$. 
We shall require the following technical lemma.

LеммA 3.3. Let $\mathbf{A}$ be a finite subdirectly irreducible algebra with Abelian monolith $\mu$. If $\operatorname{var}(\mathbf{A})$ is congruence modular, then $|\widehat{A}| \leqslant|A|$ with equality holding if and only if $\mu=(0: \mu)$.

Sketch of Proof: Let $C_{1}, \ldots, C_{m}$ be an enumeration of the $(0: \mu)$-classes of A. Each $\mu$-class is a subset of some $C_{i}$ and the different $\mu$-classes in a single $C_{i}$ have the same size. Therefore, the size of each $C_{i}$ is determined by the size $s_{i}$ and number $n_{i}$ of $\mu$-classes it contains. This implies that $|A|=\sum_{i=1}^{m}\left|C_{i}\right|=\sum_{i=1}^{m} n_{i} s_{i}$.

Now, in $\mathbf{A} \times_{\mu} \mathbf{A}$ we have $(0: \mu)_{0}=\left(0: \mu_{0}\right)$ and that each $(0: \mu)_{0}$-class is of the form $C_{i} \times_{\mu} C_{i}$ for a uniquely chosen $i$. Each such $(0: \mu)_{0}$-class contains $n_{i}$ different $\mu_{0}$-classes and they are of size $s_{i}^{2}$. Using this, one calculates that

$$
\left|A \times_{\mu} A\right|=\sum_{i=1}^{m} n_{i} s_{i}^{2}
$$

Each $(0: \mu)_{0}$-class $C$ is a union of $\Delta_{\mu,(0: \mu)}$-classes and, by examining $\Delta_{\mu,(0: \mu)}$ on $C$, one can show that for a single $\mu_{0}$-class $D \subseteq C$ we have that every element of $C$ is $\Delta_{\mu,(0: \mu)}$-related to some element of $D$ and that $D$ intersects exactly $s_{i}$ of the $\Delta_{\mu,(0: \mu)^{-}}$ classes. Hence, $\left|C / \Delta_{\mu,(0: \mu)}\right|=s_{i}$. This yields

$$
|\widehat{A}|=\sum_{i=1}^{m} s_{i} \leqslant \sum_{i=1}^{m} n_{i} s_{i}=|A| .
$$

Furthermore, equality holds if and only if each $n_{i}=1$, which means exactly that $\mu=(0: \mu)$.

LеммA 3.4. Let $\mathbf{A}$ be a subdirectly irreducible algebra which generates a congruence modular variety. If the monolith $\mu$ of $\mathbf{A}$ is $A$ belian, then $\mathbf{A}$ is normal if and only if

(i) $\mu=(0: \mu)$ and

(ii) $\mu$ is the kernel of a retraction.

Proof: By Lemma 3.2, both (i) and (ii) of this lemma hold in the normalisation of $\mathbf{A}$, hence in $\mathbf{A}$. We shall argue that if (i) and (ii) hold, then $\mathbf{A}$ is isomorphic to its normalisation.

Let $\rho: \mathbf{A} \rightarrow \mathbf{A}$ be a retraction of $\mathbf{A}$ with kernel $\mu$. Define a homomorphism

$$
\psi: \mathbf{A} \times_{\mu} \mathbf{A} \rightarrow \mathbf{A} \times_{\mu} \mathbf{A} \times_{\mu} \mathbf{A}:(a, b) \mapsto(a, b, \rho(a))=(a, b, \rho(b)) .
$$

If $d(x, y, z)$ is a difference term for $\operatorname{var}(\mathbf{A})$, then by Proposition 5.7 we have that

$$
d: \mathbf{A} \times_{\mu} \mathbf{A} \times_{\mu} \mathbf{A} \rightarrow \mathbf{A}:(a, b, c) \mapsto d(a, b, c)
$$


is a homomorphism. The composite $d \psi: \mathbf{A} \times{ }_{\mu} \mathbf{A} \rightarrow \mathbf{A}$ is easily checked to be a surjective homomorphism with kernel $\Delta_{\mu, \mu}$. Hence,

$$
\mathbf{A} \cong\left(\mathbf{A} \times{ }_{\mu} \mathbf{A}\right) / \Delta_{\mu, \mu}
$$

Since $\mu=(0: \mu)$, the algebra on the right hand side is the normalisation of $\mathbf{A}$. This finishes the proof.

Somewhat surprisingly, condition (i) of Lemma 3.4 is extraneous. That is, in a congruence modular variety, a subdirectly irreducible with Abelian monolith is normal if and only if its monolith is the kernel of a retraction. This fact is a consequence of the following lemma (whose proof does not require congruence modularity).

LEMMA 3.5. Let A be a subdirectly irreducible algebra with monolith $\mu$. If $\mu$ is the kernel of a retraction, then $(0: \mu) \leqslant \mu$.

Proof: Assume otherwise that $\mu$ is the kernel of the retraction $\rho: \mathbf{A} \rightarrow \mathbf{A}$ and that $\mu<(0: \mu)$. Let $\mathbf{B}=\rho(\mathbf{A})$. Since $\operatorname{ker} \rho=\mu<(0: \mu)$, there exists $(c, d) \in$ $\left.(0: \mu)\right|_{\mathbf{B}}-0_{\mathbf{B}}$. Choose $b \in B$ such that $|b / \mu|>1$ and then pick $a \in A-B$ such that $(a, b) \in \mu$. Since

$$
\mu=\mathbf{C g}^{\mathbf{A}}(a, b) \leqslant \mathbf{C g}^{\mathbf{A}}(c, d),
$$

there is a Mal'cev chain connecting $a$ to $b$ by polynomial images of $\{c, d\}$. Since $b \in B$ and $a \notin B$, this implies the existence of a polynomial $p \in \operatorname{Pol}_{1} A$ such that $p(c)=u \in B$ and $p(d)=v \notin B$ or the same with $c$ and $d$ interchanged. Assume that $p(\boldsymbol{x})=t^{\mathbf{A}}\left(\boldsymbol{x}, w_{1}, \ldots, w_{n}\right)$ where $t$ is a term and $w_{i} \in A$. Applying $\rho$ to the equality $t^{\mathbf{A}}\left(c, w_{1}, \ldots, w_{n}\right)=u$ (and using $\rho(c)=c, \rho(u)=u$ ) yields

$$
t^{\mathbf{A}}\left(\underline{c}, \rho\left(w_{1}\right), \ldots, \rho\left(w_{n}\right)\right)=t^{\mathbf{A}}\left(\rho(c), \rho\left(w_{1}\right), \ldots, \rho\left(w_{n}\right)\right)=\rho(u)=u=t^{\mathbf{A}}\left(\underline{c}, w_{1}, \ldots, w_{n}\right)
$$

Now, using that $(c, d) \in(0: \mu)$ and $\left(w_{i}, \rho\left(w_{i}\right)\right) \in \operatorname{ker} \rho=\mu$ we can change the $\underline{c}$ to $d$ to get

$$
t^{\mathbf{A}}\left(d, \rho\left(w_{1}\right), \ldots, \rho\left(w_{n}\right)\right)=t^{\mathbf{A}}\left(d, w_{1}, \ldots, w_{n}\right)=v
$$

But this is impossible! We have $d, \rho\left(w_{i}\right) \in B$, so

$$
v=t^{\mathbf{A}}\left(d, \rho\left(w_{1}\right), \ldots, \rho\left(w_{n}\right)\right)=t^{\mathbf{B}}\left(d, \rho\left(w_{1}\right), \ldots, \rho\left(w_{n}\right)\right) \in B
$$

and yet we chose $v=p(d) \in A-B$. This ends the proof.

The argument just given establishes a more general (and more technical) result than we claimed. Although we see no use for the more general result now, we include its statement for completeness: if $\mathbf{A}$ is an arbitrary algebra, $\rho: \mathbf{A} \rightarrow \mathbf{A}$ is an arbitrary retraction, $\mu=\operatorname{ker} \rho, B=\rho(A)$ and $\theta=\operatorname{Cg}^{A}\left(\left.(0: \mu)\right|_{B}\right)$ (that is, $\theta$ is the extension 
of the contraction of $(0: \mu))$, then $\mathbf{B}^{\theta}=\mathbf{B}$. In the case of the lemma, $\mathbf{A}$ is subdirectly irreducible with monolith $\mu$. In this case, $\mu<(0: \mu)$ implies $\mu \leqslant \theta$ which leads to the contradiction

$$
\mathbf{A}=\mathbf{B}^{\mu} \leqslant \mathbf{B}=\mathbf{B} .
$$

We connect the foregoing with the Frattini congruence and critical algebras.

Definition 3.6: If $\mathbf{A}$ is an algebra, $\mathbf{B}$ is a subalgebra of $\mathbf{A}$ and $\theta$ is a congruence on $\mathbf{A}$, then we say that $\mathbf{B}$ contains $\theta$ if $\mathbf{B}^{\theta}=\mathbf{B}$. Let $\Phi_{\mathbf{A}}$ be the join of all congruences $\theta$ which are contained in all maximal proper subalgebras of $\mathbf{A} . \Phi_{\mathbf{A}}$ is the Frattini congruence of $\mathbf{A}$.

It is easy to see that $\Phi_{A}$ is the largest congruence contained in all maximal proper subalgebras of $\mathbf{A}$. Let's call a congruence $\theta$ on $\mathbf{A}$ non-generating if

$$
\mathbf{B}^{\boldsymbol{\theta}}=\mathbf{A} \Longrightarrow \mathbf{B}=\mathbf{A}
$$

whenever $\mathbf{B}$ is a subalgebra of $\mathbf{A}$. It is straightforward to see that the Frattini congruence majorises every non-generating congruence and, when $\mathbf{A}$ is finitely generated, the Frattini congruence is the largest non-generating congruence.

THEOREM 3.7. Let $\mathbf{A}$ be a finite subdirectly irreducible algebra which generates a congruence modular variety. Assume that the monolith $\mu$ of $\mathrm{A}$ is Abelian. The following implicatons hold among the conditions enumerated below: (i) $\Longleftrightarrow$ (ii) $\Longrightarrow$ (iii) $\Longrightarrow$ (iv).

(i) A has trivial Frattini congruence.

(ii) $\mu$ is the kernel of a retraction.

(iii) $\mu=(0: \mu)$.

(iv) $\mathbf{A}$ is critical.

Proof: We shall argue that (ii) $\Longrightarrow$ (i) $\Longrightarrow$ (ii) $\Longrightarrow$ (iii) $\Longrightarrow$ (iv).

Assume (ii) holds. Since $\mu$ is the kernel of a retraction it cannot be a non-generating congruence. For suppose that $\rho: \mathbf{A} \rightarrow \mathbf{A}$ is a retraction with $\rho(\mathbf{A})=\mathbf{B}$ and $\mu=\operatorname{ker} \rho$. Then $\mathbf{B}^{\mu}=\mathbf{A}$ even though $\mathbf{B} \neq \mathbf{A}$. Hence, when (ii) holds, the Frattini congruence is not above $\mu$. This proves (i). (Here is a different argument: Since $\mathbf{A}$ is finite and $\mu$ is a minimal Abelian congruence, Theorem 2.1 of $[4]$ can be used to show that $B:=\rho(A)$ is a maximal subalgebra. $B$ does not contain $\mu$, so $\mu \not \leq \Phi_{\mathbf{A}}$.)

If (i) holds, then $\mu$ is not contained in some maximal proper subalgebra $\mathbf{B} \leqslant \mathbf{A}$. The congruence $\mu$ is Abelian and not contained in B, so Theorem 2.1 of [4] proves that $\left.\mu\right|_{\mathbf{B}}=0_{\mathbf{B}}$. Hence $\mathbf{B}$ is a $\mu$-transversal. It follows that $\mu$ is the kernel of a retraction onto $\mathbf{B}$.

The implication (ii) $\Rightarrow$ (iii) follows from Lemma 3.4. We now prove that if $A$ is subdirectly irreducible and $\mu=(0: \mu)$, then $\mathbf{A} \notin \operatorname{var}((\mathbf{H S}-1) \mathrm{A})$. For 
$\widehat{A}$ equal to the normalisation of $A$, we have $\widehat{A} \in \operatorname{var}(\mathbf{A})$. Therefore, to prove A $\notin \operatorname{var}((\mathbf{H S}-1) \mathbf{A})$ it will suffice to prove that $\hat{A} \notin \operatorname{var}((\mathbf{H S}-1) \mathbf{A})$. Assume otherwise that $\widehat{A} \in \operatorname{var}((\mathbf{H S}-1) \mathbf{A})$. From Lemma $3.2(\mathrm{v})$, we get that $\widehat{A}$ is the normalisation of some subdirectly irreducible algebra $\mathbf{B} \in \mathbf{H S}((\mathbf{H S}-1) \mathbf{A})=(\mathbf{H S}-1) \mathbf{A}$. But now (referring to Lemma 3.3), we have a cardinality problem:

$$
|\widehat{A}| \leqslant|B|<|A|=|\widehat{A}| \text {. }
$$

(The equality $|A|=|\widehat{A}|$ follows from the fact that $\mu=(0: \mu)$.) This contradiction concludes the proof.

The only implication in this proof which requires congruence modularity is (iii) $\Longrightarrow$ (iv).

\section{REFERENCES}

[1] R.M. Bryant, 'On S-critical groups', Quart. J. Math. Oxford 22 (1971), 91-101.

[2] R. Freese and R. McKenzie, 'Commutator theory for congruence modular varieties', London Math. Soc. Lecture Note Ser. 125 (1987).

[3] D. Hobby and R. McKenzie, The structure of finite algebras, Contemporary Mathematics (American Mathematics Society, Providence, Rhode Island, 1988).

[4] K.A. Kearnes, 'A Hamiltonian property for nilpotent algebras', Algebra Universalis (to appear).

[5] E.W. Kiss and S.M. Vovsi, 'Critical algebras and the Frattini congruence', Algebra Universalis (to appear).

[6] I.D. Macdonald, 'A theorem on critical p-groups', in Proc. Intern. Conf. Theory of Groups, Australian National University, Canberra 1965 (Gordon and Breach, 1967).

[7] Yu. M. Malt'sev, 'Cross varieties of algebras', DAN SSSR 278 (1984), 1060-1063.

[8] H. Neumann, Varieties of groups (Springer-Verlag, Berlin, Heidelberg, New York, 1967).

[9] S.M. Vovsi, 'On locally finite varieties of group representations', Izv. Vyssh. Uchebn. Zaved. Mat. 6 (1972), 16-27.

[10] S.M. Vovsi, 'On critical multioperator groups', Sibirsk. Mat. Zh. 30 (1989), 213-215.

\footnotetext{
Department of Mathematical Sciences

University of Arkansas

Fayetteville AR 72701

United States of America
} 